\section{Gamma-Rays from Thulium-170}

IN order to verify that the gamma-rays from thulium-170 reported ${ }^{1}$, in addition to the wellestablished transition of $\sim 83 \mathrm{keV}$., were not, in fact, Bremsstrahlung ${ }^{2}$, the secondary electron spectrum from a source of 5-10-millicuries strength has been examined in the short-lens beta-ray spectrometer using high transmission (low resolving power).

Thin gold and both thick and thin lead radiators were used, and in each case a weak $K$ photo-electron line from a gamma-ray of energy $198 \pm 2 \mathrm{keV}$. was observed. Measurements were also made on the continuous spectrum of Compton electrons ejected from the brass capsule containing the active material. A group with an end-point corresponding to a gammaray energy of $360 \pm 10 \mathrm{keV}$. can be identified with some certainty, and there is also a group of higher energy and much lower (1) intensity. If this is a Compton eléctron group, its shape implies the presence of at least two gamma-rays, of which the harder has an energy of $\sim 550 \mathrm{keV} \cdot( \pm 40)$. The other possibility is that the upper group is due to Bremsstrahlung.

Cavendish Laboratory,

$$
\text { P. J. GRANT }
$$

\section{Cambridge.}

Feb. 28.

${ }^{1}$ Grant, P. J., and Richmond, R., Proc. Phys. Soc., A, 62, 573 (1949) 2 Graham, R. L., and Tomlin, D. H., Nature, 164, 278 (1949).

\section{Sudden Commencements in Geomagnetism}

From a recent study of geomagnetic 'sudden commencements' at seven magnetic observatories, Ferraro and Parkinson ${ }^{1}$ suggested the possible dependence on magnetic longitude of the occurrence of what they termed "Type II" sudden commencements, that is, the type in which the increase in the horizontal force $H$ is preceded by a smaller preliminary movement in the opposite direction.

This suggestion has been tested by an examination of sudden commencements recorded at the Lerwick Observatory (Shetland Is.) between April 1934 and December 1949. This examination yielded a total of 340 sudden commencements. Following Ferraro and Parkinson's classification, 65 of these 340 Lerwick sudden commencements were classified Type I (characterized by a sudden increase in $H$ following a more or less quiet period), 162 were classified Type II, and 113 Type III (that is, sudden commencements of the 'inverted' type, characterized by a decrease in $H$ ). Thus the ratio of Type II to total number of sudden commencements found for Ler. wick (magnetic longitude $88.6^{\circ}$ ) was $0 \cdot 48$. This value is very close to that suggested by Ferraro and Parkinson on the basis of magnetic longitude.

Detailed examination of the Lerwick sudden commencements has yielded other interesting features which are under investigation.

We are indebted to the Director, Meteorological Office, London, for permission to publish this note.

$$
\begin{array}{cl} 
& \text { R. A. WATSON } \\
\text { Meteorological Office, } & \text { D. H. MoINTOSH } \\
\text { Edinburgh. } & \\
\text { April 25. } & \\
\text { ' Nature, 165, } 243(1950) . &
\end{array}
$$

\section{Masses of Fundamental Particles}

ThE following empirical relation

$$
\left(\frac{M_{1}}{m}\right)^{2}\left(\frac{M_{1}}{m}-\frac{M_{2}}{m}\right)=\frac{2}{\alpha^{3}}=2 \times 137^{3}
$$

has been found, connecting the masses $M_{1}$ and $M_{2}$ of the fundamental particles and the electron mass $m$.

Substituting for $M_{2}$ the present value $1837.56 \mathrm{~m}$ for the mass of the neutral hydrogen atom, it is found that $M_{1}$ is $1839.08 \mathrm{~m}$, in excellent agreement with the experimental neutron mass $1839.05 \mathrm{~m}$. This simple relation between the neutron and hydrogen atom is consistent with Eddington's $\mathrm{s}^{1}$ concept of the neutron as a hydrogen atom in the 0 -quantum state.

In the same way, substituting $M_{2}=216 \mathrm{~m}$ for the mass of the $\mu$-meson, it is found that $M_{1}=281 \mathrm{~m}$, in good agreement with the present experimental value of $285 \pm 5 \mathrm{~m}$ for the $\pi$-meson.

Assuming the existence of a neutrino of zero restmass, it is seen that $M_{1}=173 \mathrm{~m}$; this value is in close agreement with Eddington's ${ }^{1}$ value of $173.98 \mathrm{~m}$ for the meson mass. No derivation of this relation has yet been found.

\section{G. STEPRENSON}

Department of Physics,

Imperial College of Science and Technology, London, S.W.7.

Feb. 22.

1 Eddington, A. S., "Fundamental Theory" (Cambridge, 1948).

\section{Mechanism of the Geiger Discharge}

IN the original theory of Townsend, the discharge phenomena in the Geiger counter are explained on the assumption of ionization by electrons ( $\alpha$ ions per cm.) followed by an ionization by positive ions ( $\beta$ ions per $\mathrm{cm}$.). Later this theory, especially as a consequence of the fact that ionization by positive ions requires a high energy, was modified in such a way that the secondary process was considered to consist of electrons liberated from the cathode by photons from the primary avalanche $(\theta n g$ photoelectrons from one field electron per cm. path). Both theories give analogous mathematical expressions for the sparking equation and for the current amplification in the homogeneous field between plane surfaces. Thus it is impossible to decide between the two possibilities.

By the calculation of the avalanches in the axial. symmetrical field of the Geiger counter, it can be proved, however, that the observed values of the starting voltage $V_{A}$ are best explained by assuming ionization by positive ions. The Geiger discharge, in contrast to the current idea of photo-electric multiplication, is thus explained as being started by a small primary electron avalanche, from which positive ions produce new ion-pairs in the immediate neighbourhood of the wire. Electrons from these new ion-pairs form secondary avalanches in which secondary positive ions are produced in a quantity which at the starting voltage is equal to the number of ions in the primary avalanche.

This assumption leads to the following sparking equation at $V_{\boldsymbol{A}}$ :

$$
\int_{r i}^{r e}\left(M_{r}-1\right) \beta d r=1,
$$

